\title{
Analysis of IUE spectra of helium-rich white dwarf stars ${ }^{\star}$
}

\author{
B. G. Castanheira ${ }^{1}$, S. O. Kepler ${ }^{1}$, G. Handler ${ }^{2,3}$, and D. Koester ${ }^{4}$ \\ 1 Instituto de Física, Universidade Federal do Rio Grande do Sul, 91501-900 Porto-Alegre, RS, Brazil \\ e-mail: barbara@if.ufrgs.br \\ 2 Institut für Astronomie, Universität Wien, Türkenschanzstrasse 17, 1180, Wien, Austria \\ 3 South African Astronomical Observatory, PO Box 9, Observatory 7935, South Africa \\ 4 Institut für Theoretische Physik und Astrophysik, Universität Kiel, 24098 Kiel, Germany
}

Received 17 September 2005 / Accepted 30 December 2005

\section{ABSTRACT}

We studied the class of DB white dwarf stars, using re-calibrated UV spectra for thirty four DBs obtained with the IUE satellite. By comparing the observed energy distributions with model atmospheres, we simultaneously determine spectroscopic distances $(d)$, effective temperature $\left(T_{\text {eff }}\right)$, and surface gravities $(\log g)$. Using parallax measurements and previous determinations of $T_{\text {eff }}$ and $\log g$ from optical spectra, we can study whether the atmospheres of eleven DB stars are consistent with pure He or have a small amount of $\mathrm{H}$ contamination. We also report on our observations of seventeen stars with $T_{\text {eff }}$ close to the DB instability strip through time series photometry and found them to be non variable within our detection limits.

Key words. stars: white dwarfs - stars: variables: general - stars: oscillations - ultraviolet: stars

\section{Introduction}

Among all known white dwarf stars, around $20 \%$ have a helium $(\mathrm{He})$ dominated atmosphere, and are thus assigned the spectral type DB. Most of these stars are believed to be result of the born again or a very late He thermal pulse during the early planetary nebula cooling phase (e.g. Althaus et al. 2005). In this event, the residual hydrogen $(\mathrm{H})$ is completely burnt, the star returns quickly to AGB phase and again to planetary nebula, this time, without $\mathrm{H}$. As they stars cool down, DBs cross an instability strip, where they are seen as multi-periodic pulsators. Beauchamp et al. (1999) determined its boundaries as $27800 \geq T_{\text {eff }} \geq 22400 \mathrm{~K}$ from a comparison of their pure He model atmosphere grid with ML2 $/ \alpha=1.25$ to optical spectra, and $24700 \geq T_{\text {eff }} \geq 21800 \mathrm{~K}$, if undetectable traces of hydrogen $(\mathrm{H})$ are allowed in the models.

The study of the instability strip of the DBs is still a challenge because of the small number of known pulsators; only seventeen are known to date (Nitta et al. 2005). Another difficulty is that the determinations of $T_{\text {eff }}$ and $\log g$ from spectra are degenerate as, in general, these two parameters are correlated. Working with optical spectra is even more problematic, as possible contamination with even trace amounts of hydrogen that are undetected in the spectra can decrease the resulting effective temperatures by up to $3000 \mathrm{~K}$ and $\log g$ by up to 0.05 dex (Beauchamp et al. 1999). The uncertainty in $T_{\text {eff }}$

^ Partially based on observations at Observatório do Pico dos Dias/ LNA. derived from the published optical spectra is thus comparable to the width of the instability strip.

The DBs have been studied since the $1960 \mathrm{~s}$, but especially after the discovery of a pulsator, GD 358, based on theoretical predictions (Winget et al. 1982). This star is the brightest and one of the best studied variable $\mathrm{He}$ atmosphere white dwarf (DBV) stars. Because pulsation theory gives detailed predictions of DBV properties, these stars can be used to study neutrino rates probing the electro-weak theory (Winget et al. 2004; Córsico \& Althaus 2004), the $\mathrm{C}(\alpha, \gamma) \mathrm{O}$ cross section (Metcalfe 2003, 2005), and the $\mathrm{He}^{3} / \mathrm{He}^{4}$ separation (Wolff et al. 2002; Montgomery \& Winget 2000) which cannot be achieved in any terrestrial laboratory. Pulsations in DBs are predicted to exist in a narrow temperature range, $\sim 3000 \mathrm{~K}$ wide, but it has been difficult to measure $T_{\text {eff }}$ with sufficient accuracy to determine the edges of the instability strip.

Considerable interest is focused on the accurate determination of atmospheric parameters for DB white dwarfs, for yet another reason, the so called "DB gap", where there are no observed DB stars. It occurs between 45000 and $30000 \mathrm{~K}$ in the cooling sequence (e.g. Hansen \& Liebert 2003). The physical reason of the DB gap is still not understood. However, many theories attempt to explain why there would be no DBs within this range of temperature. One possibility is that DBs would turn into DAs (white dwarf with pure $\mathrm{H}$ atmospheres) by dragging $\mathrm{H}$ to the surface of the star, blocking the atmosphere. In this scenario, we expect to find more $\mathrm{H}$ in the hot DBs than 
in the cooler ones. We also investigate that possibility in this paper, but we do not confirm this theory.

\section{Fitting the ultraviolet spectra}

To study the DBs as a class and the characteristics of their instability strip, we used ultraviolet spectra because they are less affected by possible trace amounts of $H$ that plague the optical determination of the effective temperature (Beauchamp et al. 1999). The data we use to determine the distance $(d)$, effective temperature $\left(T_{\text {eff }}\right)$, and surface gravity $(\log g)$ are the recalibrated ultraviolet spectra for DB stars, obtained with the International Ultraviolet Explorer (IUE) satellite and published by Holberg et al. (2003). The spectra were re-calibrated with the New Spectroscopic Image Processing System (NEWSIPS) data reduction by NASA, and in the low-dispersion spectral mode with a resolution of $\sim 6 \AA$.

One of the major motivations to use the archive of IUE lowdispersion spectra, besides it comprising an homogeneous sample, is to work with spectra of which the absolute calibration is based on a synthetic model atmosphere energy distribution for the white dwarf star G191-B2B (WD 0501+527). The models we fit are the same kind used in the flux calibration.

We used a new grid of Koester's model atmospheres, with input physics and methods similar to those described in Finley et al. (1997), consisting of models with $T_{\text {eff }}$ from $12000 \mathrm{~K}$ to $28000 \mathrm{~K}$, and a step of $500 \mathrm{~K}$, and $\log g$ from 7.0 to 9.0 , with 0.1 dex step. We used two sets of model atmospheres: pure $\mathrm{He}$ and $\mathrm{He}$ contaminated with a small amount of $\mathrm{H}$ $[\log y \equiv \log (N \mathrm{He} / N \mathrm{H})=-3.0]$. This is the the upper limit for the amount of $\mathrm{H}$ contamination for a star not show discernible $\mathrm{H}$ lines in the optical spectra, i.e., to be classified as a DB and not as a DBA. All models were calculated with ML2 $/ \alpha=0.6$ mixing length theory, considering that Bergeron et al. (1995) and Koester \& Vauclair (1997) have shown this choice of mixing length gives consistent results in the UV and optical, for the DAs. There is no reason to expect the mixing length description to be different for DBs. These models were used to simultaneously fit $T_{\text {eff }}, \log g$ and $d$ to the available IUE spectra.

We calculated the minima in $\chi^{2}$ between the observed spectra and the models, allowing the three parameters, $T_{\text {eff }}, \log g$, and $d$, to vary. We used the model radii described in Althaus \& Benvenuto (1997), available in http://www.fcaglp.unlp.edu.ar/evolgroup/ tracks.html.

Our determinations of $T_{\mathrm{eff}}, \log g$ and the distance for all DB stars with IUE spectra available are shown in Table 1. In Cols. 3-5, we show the values derived using pure He models, and in Cols. 6-8, the same parameters using $\mathrm{He} / \mathrm{H}$ models.

\section{Comparison of results}

Our determinations are still degenerate with respect to the contamination of $\mathrm{H}$ in the He atmosphere. To minimize this effect, we used external measurements, like: optical spectra, parallax measurements, and $V$ magnitudes, if available.

\subsection{Distance moduli}

To test the reliability of our spectroscopic distances, we used our determinations of $T_{\text {eff }}$ and $\log g$, Bergeron's et al. (2001) absolute magnitude, and the published $V$ magnitude to estimate the distance moduli. In Table 2 we show the derived distances from this method and the distances after cross correlating both solutions.

In almost all cases, both spectroscopic and magnitude derived distances agree, even though we used independent model grids.

\subsection{Parallax measurements}

For six stars of our sample, parallax measurements are available (van Altena et al. 2001). Comparing these distances with the ones derived spectroscopically, the better agreement, in general, is the solution derived using pure He models. There are two stars, GD 358 and GD 408, for which we could not distinguish the atmospheric composition. Feige 4 is an exception, for which both spectroscopic solutions do not agree with the published parallax. However, this is the faintest star in our sample with parallax measurement, with magnitude close to the limit of the catalog. In Table 3, we show the parallax distance and the best stellar composition cross correlating the solutions.

The IUE spectra of Feige 4 (full line) is shown in Fig. 1 in comparison to the models. The best models derived from the spectra are with $T_{\text {eff }}=19000 \mathrm{~K}, \log g=8.50, d=61 \mathrm{pc}$, and pure He (dashed line) and with $T_{\text {eff }}=18000 \mathrm{~K}, \log g=7.50$, $d=112 \mathrm{pc}$, and $\mathrm{He} / \mathrm{H}$ grid (dotted line). Using the distance derived from parallax, $d=33 \mathrm{pc}$, the best models are not only much cooler, $T_{\text {eff }}=14000 \mathrm{~K}$ for pure He models (dotteddashed line) and $T_{\text {eff }}=17000 \mathrm{~K}$ for He/H models (long dashed line), but they also do not fit the slope of the observed spectra. Another argument to claim the parallax measurement is not correct is that this star has apparent magnitude $V=15.3$, too faint for such a large parallax, unless the radius is extremely small, i.e., high mass, incompatible with the observed spectra.

\subsection{Comparison with optical spectra results}

Beauchamp et al. (1999) studied the optical spectra of eight known DBVs together with fifteen other DB and DBA stars with temperatures above $20000 \mathrm{~K}$. For DBs, including DBVs, they used a pure $\mathrm{He}$ atmosphere composition, or a homogeneous $\mathrm{H} / \mathrm{He}$ ratio with only traces of $\mathrm{H}$, at the detection threshold - defined as that which would produce barely visible $\mathrm{H} \beta$ or $\mathrm{H} \gamma$ features, two lines included in their spectra. The influence of small, spectroscopically invisible amounts of $\mathrm{H}$ in the DB's atmospheres is an important issue in the definition of the temperature scale in the optical, because $T_{\text {eff }}$ determined using $\mathrm{He}$ models with small admixture of $\mathrm{H}$ are often lower by a few thousand of $\mathrm{K}$, than those determined with pure He models.

The instability strip Beauchamp et al. (1999) derived from the analysis of optical spectra contains non-variable stars. Its $T_{\text {eff }}$ is also uncertain due to the possible presence of trace amounts of $\mathrm{H}$ in the stellar atmospheres. In Table 4, we compare our determination for $T_{\text {eff }}$ from UV spectra, described 
Table 1. Atmospheric parameters and distance determined from IUE spectra, using pure He models (He) and He contaminated with a small amount of $\mathrm{H}(\mathrm{He} / \mathrm{H})$ models. An asterisk indicates a DBA star, for which our determinations are not adequate.

\begin{tabular}{|c|c|c|c|c|c|c|c|}
\hline Name & WD & He $T_{\text {eff }}(\mathrm{K})$ & He $\log g$ & $\mathrm{He} d(\mathrm{pc})$ & $\mathrm{He} / \mathrm{H} T_{\text {eff }}(\mathrm{K})$ & $\mathrm{He} / \mathrm{H} \log g$ & $\mathrm{He} / \mathrm{H} d(\mathrm{pc})$ \\
\hline G 266-32 & $0000-170$ & $16000 \pm 600$ & $8.50 \pm 0.60$ & $39 \pm 12$ & $14000 \pm 270$ & $8.00 \pm 0.02$ & $46 \pm 3$ \\
\hline GD 408 & $0002+729$ & $14000 \pm 40$ & $8.50 \pm 0.04$ & $26 \pm 1$ & $14000 \pm 40$ & $7.50 \pm 0.07$ & $54 \pm 2$ \\
\hline Feige 4 & $0017+136$ & $19000 \pm 170$ & $8.00 \pm 0.17$ & $61 \pm 5$ & $18000 \pm 60$ & $7.50 \pm 0.06$ & $112 \pm 7$ \\
\hline G 270-124 & 0100-068 & $20500 \pm 130$ & $8.40 \pm 0.23$ & $34 \pm 4$ & $19000 \pm 100$ & $7.00 \pm 0.15$ & $69 \pm 5$ \\
\hline PG 0112+104 & $0112+104$ & $27000 \pm 110$ & $7.50 \pm 0.03$ & $136 \pm 2$ & $27000 \pm 130$ & $8.50 \pm 0.06$ & $71 \pm 2$ \\
\hline GD 40 & 0300-013 & $15000 \pm 420$ & $7.50 \pm 0.21$ & $104 \pm 11$ & $15000 \pm 310$ & $8.50 \pm 0.16$ & $58 \pm 5$ \\
\hline BPM 17088 & $0308-565$ & $21500 \pm 190$ & $7.70 \pm 0.08$ & $58 \pm 2$ & $21000 \pm 280$ & $8.00 \pm 0.14$ & $49 \pm 3$ \\
\hline BPM 17731 & 0418-539 & $20000 \pm 140$ & $8.00 \pm 0.14$ & $83 \pm 6$ & $19000 \pm 110$ & $7.50 \pm 0.06$ & $110 \pm 3$ \\
\hline BPM 18164 & 0615-591 & $16000 \pm 50$ & $8.50 \pm 0.17$ & $26 \pm 2$ & $16000 \pm 40$ & $7.00 \pm 0.04$ & $64 \pm 1$ \\
\hline Ton 10 & $0840+262$ & $21000 \pm 90$ & $7.50 \pm 0.09$ & $96 \pm 4$ & $18000 \pm 100$ & $7.00 \pm 0.15$ & $103 \pm 8$ \\
\hline L748-70 & 0845-188 & $18000 \pm 140$ & $7.50 \pm 0.14$ & $129 \pm 9$ & $18000 \pm 60$ & $7.50 \pm 0.06$ & $135 \pm 4$ \\
\hline PG $0853+163^{*}$ & $0853+163$ & $21000 \pm 450$ & $7.70 \pm 0.18$ & $126 \pm 11$ & $20000 \pm 650$ & $7.50 \pm 0.65$ & $137 \pm 44$ \\
\hline PG $0948+013$ & $0948+013$ & $19000 \pm 280$ & $8.20 \pm 0.19$ & $92 \pm 9$ & $18000 \pm 150$ & $7.00 \pm 0.15$ & $173 \pm 13$ \\
\hline GD 303 & $1011+570$ & $18000 \pm 140$ & $7.50 \pm 0.14$ & $75 \pm 5$ & $18000 \pm 60$ & $7.50 \pm 0.03$ & $78 \pm 1$ \\
\hline PG $1115+158$ & $1115+158$ & $23000 \pm 500$ & $8.50 \pm 0.10$ & $137 \pm 7$ & $22000 \pm 500$ & $7.00 \pm 0.25$ & $321 \pm 40$ \\
\hline PG 1149-133* & $1149-133$ & $20500 \pm 440$ & $7.60 \pm 0.57$ & $161 \pm 46$ & $19000 \pm 260$ & $7.00 \pm 0.19$ & $196 \pm 19$ \\
\hline PG $1311+129^{*}$ & $1311+129$ & $26500 \pm 450$ & $7.70 \pm 0.05$ & $249 \pm 6$ & $27000 \pm 280$ & $7.50 \pm 0.14$ & $298 \pm 14$ \\
\hline PG 1326-037 & $1326-037$ & $21500 \pm 290$ & $8.40 \pm 0.35$ & $81 \pm 14$ & $20000 \pm 100$ & $8.00 \pm 0.10$ & $100 \pm 5$ \\
\hline GD 325 & $1333+487$ & $16000 \pm 40$ & $8.20 \pm 0.01$ & $34 \pm 0.2$ & $15000 \pm 120$ & $7.00 \pm 0.06$ & $61 \pm 2$ \\
\hline PG $1351+489$ & $1351+489$ & $22500 \pm 190$ & $7.60 \pm 0.15$ & $194 \pm 14$ & $22000 \pm 150$ & $7.00 \pm 0.07$ & $266 \pm 10$ \\
\hline PG $1411+218$ & $1411+218$ & $15000 \pm 70$ & $7.80 \pm 0.01$ & $49 \pm 0.3$ & $14000 \pm 70$ & $7.00 \pm 0.03$ & $66 \pm 1$ \\
\hline G 200-39 & $1425+540$ & $15000 \pm 110$ & $7.70 \pm 0.09$ & $74 \pm 3$ & $15000 \pm 310$ & $8.50 \pm 0.31$ & $47 \pm 7$ \\
\hline PG $1445+152$ & $1445+152$ & $21500 \pm 120$ & $8.40 \pm 0.12$ & $91 \pm 5$ & $21000 \pm 120$ & $8.50 \pm 0.06$ & $86 \pm 3$ \\
\hline PG $1456+103^{*}$ & $1456+103$ & $24000 \pm 190$ & $8.50 \pm 0.27$ & $110 \pm 15$ & $24000 \pm 290$ & $9.00 \pm 0.14$ & $76 \pm 5$ \\
\hline G 256-18 & $1459+821$ & $16000 \pm 50$ & $8.00 \pm 0.03$ & $53 \pm 1$ & $15000 \pm 310$ & $7.00 \pm 0.05$ & $83 \pm 2$ \\
\hline GD 190 & $1542+182$ & $22500 \pm 90$ & $8.50 \pm 0.11$ & $48 \pm 3$ & $21000 \pm 60$ & $7.00 \pm 0.06$ & $106 \pm 3$ \\
\hline GD 358 & $1645+325$ & $24500 \pm 130$ & $8.50 \pm 0.10$ & $29 \pm 1$ & $24000 \pm 50$ & $8.50 \pm 0.03$ & $30 \pm 0.4$ \\
\hline PG $1654+160$ & $1654+160$ & $25000 \pm 550$ & $7.50 \pm 0.11$ & $237 \pm 13$ & $26000 \pm 1100$ & $7.00 \pm 0.55$ & $331 \pm 91$ \\
\hline L 7-44 & $1708-871$ & $23000 \pm 610$ & $8.30 \pm 0.42$ & $55 \pm 12$ & $21000 \pm 680$ & $7.00 \pm 0.34$ & $106 \pm 18$ \\
\hline GD 378 & $1822+410$ & $17000 \pm 60$ & $8.20 \pm 0.04$ & $39 \pm 1$ & $16000 \pm 80$ & $7.00 \pm 0.08$ & $70 \pm 3$ \\
\hline L 1573-31 & $1940+374$ & $17000 \pm 50$ & $7.60 \pm 0.05$ & $62 \pm 1$ & $17000 \pm 40$ & $7.00 \pm 0.07$ & $86 \pm 3$ \\
\hline BPM 26944 & $2034-532$ & $17000 \pm 390$ & $8.50 \pm 0.39$ & $34 \pm 7$ & $17000 \pm 80$ & $7.00 \pm 0.12$ & $86 \pm 5$ \\
\hline G 26-10 & $2129+000$ & $13000 \pm 60$ & $7.50 \pm 0.06$ & $50 \pm 2$ & $13000 \pm 40$ & $7.50 \pm 0.02$ & $54 \pm 1$ \\
\hline LTT 9031 & 2224-344 & $19000 \pm 160$ & $7.50 \pm 0.16$ & $72 \pm 6$ & $18000 \pm 130$ & $7.00 \pm 0.13$ & $88 \pm 6$ \\
\hline
\end{tabular}

in Sect. 2, with those derived from optical spectra. The optical spectra also give two solutions, with or without trace $\mathrm{H}$. For seven DB stars, the best agreement in $T_{\text {eff }}$ in both UV and optical range is for atmospheres consistent with a small amount of $\mathrm{H}$ instead of none. The exception is the star GD 358, which has a higher probability of having a pure He atmosphere in agreement with Provencal et al. (2000) determination of $\log (N \mathrm{He} / N \mathrm{H}) \leq-5$ for this star. In Fig. 2, we show a comparison between UV ( $x$-axis) and optical ( $y$-axis) spectroscopic determinations of $T_{\text {eff }}$, for a pure $\mathrm{He}$ atmosphere and a $\mathrm{He} / \mathrm{H}$ atmosphere. The closer a given data point is to the dashed line (1:1 correspondence between UV and optical spectra), the better the solution for the atmosphere composition becomes. The dotted lines link the two atmosphere determinations for a given star, showing that $\mathrm{He} / \mathrm{H}$ atmospheres are more likely for this sample.
GD 358 is the only star in our sample which both parallax measurement and optical spectra determination is available. We cannot distinguish the best atmosphere composition from the parallax, but a pure He atmosphere is still consistent with the optical spectra determination.

For the star GD 190, even though we get a better agreement with the optical spectra for a contaminated atmosphere, Provencal et al. (2000) obtained an upper limit of $\log (N \mathrm{He} / N \mathrm{H}) \leq-6.5$, consistent with a pure He atmosphere.

An important consideration is that we fitted all stars using DB models, never with DBA models. From the IUE spectra, we cannot determine if a star is a DBA or not. The optical spectra of PG $0853+163$, PG 1149-133, PG 1311+129, and PG $1456+103$ do show $\mathrm{H}$, which has been taken into account by Beauchamp et al. (1999) by using models with a considerable amount of $\mathrm{H}$. Our temperatures for DBA stars are 
Table 2. Distance determined from distance modulus (Cols. 2-3) using IUE $T_{\text {eff }}$ and $\log g$, compared to absolute magnitude models and available $V$ magnitudes. We used both pure He models (He) and $\mathrm{He}$ contaminated with a small amount of $\mathrm{H}(\mathrm{He} / \mathrm{H})$ models. The last 2 columns are the distances after cross correlating these values and the spectroscopic distances (see values in Table 1).

\begin{tabular}{|c|c|c|c|c|}
\hline Name & He $d(\mathrm{pc})$ & $\overline{\mathrm{He} / \mathrm{H} d(\mathrm{pc})}$ & $\operatorname{He} d_{\text {cross }}(\mathrm{pc})$ & $\mathrm{He} / \mathrm{H} d_{\text {cross }}(\mathrm{pc})$ \\
\hline G 266-32 & $36_{-16}^{+22}$ & $45 \pm 2$ & $37_{-10}^{+13}$ & $46 \pm 2$ \\
\hline GD 408 & $26 \pm 1$ & $51 \pm 2$ & $26 \pm 1$ & $53 \pm 1$ \\
\hline Feige 4 & $81 \pm 7$ & $108_{-3}^{+4}$ & $71 \pm 4$ & $110 \pm 4$ \\
\hline G $270-124$ & $35_{-6}^{+7}$ & $88_{-9}^{+10}$ & $34 \pm 4$ & $78_{-5}^{+6}$ \\
\hline PG 0112+104 & $144_{-20}^{+28}$ & $71_{-4}^{+3}$ & $140_{-10}^{+14}$ & $71 \pm 2$ \\
\hline GD 40 & $100_{-15}^{+20}$ & $50_{-8}^{+3}$ & $102_{-9}^{+11}$ & $54_{-5}^{+3}$ \\
\hline BPM 17088 & $61_{-3}^{+4}$ & $50 \pm 5$ & $60 \pm 2$ & $50 \pm 3$ \\
\hline BPM 17731 & $82 \pm 6$ & $109_{-3}^{+4}$ & $83 \pm 4$ & $109 \pm 2$ \\
\hline BPM 18164 & $27 \pm 4$ & $79_{-9}^{+10}$ & $27 \pm 2$ & $71_{-4}^{+5}$ \\
\hline Ton 10 & $81_{-2}^{+4}$ & $104_{-8}^{+9}$ & $89_{-2}^{+3}$ & $104 \pm 6$ \\
\hline L748-70 & $122_{-12}^{+14}$ & $122_{-5}^{+6}$ & $125_{-7}^{+8}$ & $128_{-3}^{+4}$ \\
\hline PG $0853+163^{*}$ & $135_{-16}^{+18}$ & $150_{-56}^{+95}$ & $131_{-10}^{+11}$ & $144_{-36}^{+52}$ \\
\hline PG 0948+013 & $92_{-13}^{+15}$ & $205_{-23}^{+25}$ & $92_{-8}^{+9}$ & $189_{-13}^{+14}$ \\
\hline GD 303 & $119_{-11}^{+14}$ & $119_{-3}^{+7}$ & $97_{-6}^{+7}$ & $98_{-1}^{+3}$ \\
\hline PG $1115+158$ & $95_{-9}^{+8}$ & $260_{-45}^{+53}$ & $116_{-6}^{+5}$ & $291_{-30}^{+35}$ \\
\hline PG $1149-133^{*}$ & $178_{-57}^{+92}$ & $258_{-36}^{+42}$ & $169_{-37}^{+51}$ & $227_{-21}^{+23}$ \\
\hline PG $1311+129^{*}$ & $187 \pm 9$ & $218_{-22}^{+29}$ & $218 \pm 5$ & $258_{-13}^{+16}$ \\
\hline PG 1326-037 & $82_{-22}^{+23}$ & $103_{-8}^{+7}$ & $82 \pm 13$ & $101_{-5}^{+4}$ \\
\hline GD 325 & $33_{-0.3}^{+0.2}$ & $70 \pm 3$ & $33_{-0.2}^{+0.1}$ & $66 \pm 2$ \\
\hline PG $1351+489$ & $194_{-36}^{+21}$ & $293_{-15}^{+17}$ & $194_{-19}^{+13}$ & $280_{-9}^{+10}$ \\
\hline PG $1411+218$ & $48_{-0.6}^{+0.5}$ & $76 \pm 2$ & $48 \pm 0.3$ & $71 \pm 1$ \\
\hline G 200-39 & $69_{-4}^{+5}$ & $39_{-10}^{+11}$ & $72 \pm 3$ & $43_{-6}^{+7}$ \\
\hline PG $1445+152$ & $77 \pm 7$ & $71 \pm 4$ & $84 \pm 4$ & $78 \pm 2$ \\
\hline PG $1456+103^{*}$ & $87 \pm 18$ & $57_{-7}^{+8}$ & $98 \pm 12$ & $66 \pm 4$ \\
\hline G 256-18 & $60_{-2}^{+1}$ & $111 \pm 7$ & $56 \pm 1$ & $97 \pm 4$ \\
\hline GD 190 & $49_{-5}^{+4}$ & $134 \pm 6$ & $49 \pm 3$ & $120 \pm 3$ \\
\hline GD 358 & $30 \pm 2$ & $30 \pm 1$ & $30 \pm 1$ & $30_{-0.4}^{+0.3}$ \\
\hline PG $1654+160$ & $196_{-16}^{+22}$ & $302_{-113}^{+192}$ & $216_{-10}^{+13}$ & $317_{-73}^{+106}$ \\
\hline L 7-44 & $57_{-12}^{+14}$ & $133_{-22}^{+28}$ & $56_{-8}^{+9}$ & $119_{-14}^{+17}$ \\
\hline GD 378 & $42 \pm 1$ & $90 \pm 6$ & $40 \pm 1$ & $80 \pm 3$ \\
\hline L 1573-31 & $67 \pm 2$ & $103 \pm 4$ & $64 \pm 1$ & $95 \pm 3$ \\
\hline BPM 26944 & $34_{-11}^{+13}$ & $101_{-9}^{+10}$ & $34_{-6}^{+7}$ & $93_{-5}^{+6}$ \\
\hline G $26-10$ & $58_{-2}^{+3}$ & $58 \pm 1$ & $54 \pm 2$ & $56 \pm 1$ \\
\hline LTT 9031 & $78_{-8}^{+10}$ & $108_{-11}^{+12}$ & $75_{-5}^{+6}$ & $98 \pm 6$ \\
\hline
\end{tabular}

therefore not reliable, but differences in $T_{\text {eff }}$ from our models with or without trace $\mathrm{H}$ are the same order as our uncertainties.

We did not compare $\log g$ values, as their uncertainties are too large from both UV and optical spectra.

\section{Looking for new pulsators}

Robinson \& Winget (1983) reported a search for pulsating DB white dwarf stars, classifying twenty nine stars as nonvariable. Expanding this search, we acquired time-series photometric observations of another thirteen DB white dwarf stars, which have $T_{\text {eff }}$ close to the edges of the DB observed instability strip, plus one DA (H atmosphere white dwarf) during two observing runs at the South African Astronomical Observatory (SAAO), and four DBs at the Observatório Pico
Table 3. Using the distance determined by parallax measurements (second column), we study the best agreement with our fits, deriving the atmosphere composition (third column).

\begin{tabular}{ccc}
\hline \hline Star & $d(\mathrm{pc})$ & Atmosphere \\
\hline GD 408 & $35 \pm 6$ & undetermined \\
Feige 4 & $33 \pm 10$ & undetermined \\
GD 325 & $35 \pm 4$ & He/H \\
G 200-39 & $58 \pm 13$ & pure He \\
GD 358 & $37 \pm 4$ & undetermined \\
L 1573-31 & $49 \pm 7$ & pure He \\
\hline
\end{tabular}

dos Dias (OPD) in other three runs, to search for variability. At SAAO, one of us $(\mathrm{GH})$ used the $0.75-\mathrm{m}$ telescope in 
Table 4. Atmospheric parameter determinations from UV spectra in comparison to those derived by Beauchamp et al. (1999) using optical spectra. The last column shows the best agreement in atmosphere composition using both independent determinations.

\begin{tabular}{ccc|ccc}
\hline \hline Name & UV He & UV He/H & Optical He & Optical He/H & Atmosphere \\
\hline G 270-124 & $20500 \pm 130$ & $19000 \pm 100$ & 22500 & 20500 & $\mathrm{He} / \mathrm{H}$ \\
PG 0112+104 & $27000 \pm 110$ & $27000 \pm 130$ & 31500 & 28300 & $\mathrm{He} / \mathrm{H}$ \\
PG 1115+158 & $23000 \pm 500$ & $22000 \pm 500$ & 25300 & 21800 & $\mathrm{He} / \mathrm{H}$ \\
PG 1351+489 & $22500 \pm 190$ & $22000 \pm 150$ & 26100 & 22600 & $\mathrm{He} / \mathrm{H}$ \\
PG 1445+152 & $21500 \pm 120$ & $21000 \pm 120$ & 23600 & 22200 & $\mathrm{He} / \mathrm{H}$ \\
GD 190 & $22500 \pm 90$ & $21000 \pm 60$ & 21500 & 21000 & $\mathrm{He} / \mathrm{H}$ \\
GD 358 & $24500 \pm 130$ & $24000 \pm 50$ & 24900 & 24700 & $\mathrm{He}$ \\
PG 1654+160 & $25000 \pm 550$ & $26000 \pm 1100$ & 27800 & 24300 & $\mathrm{He} / \mathrm{H}$ \\
\hline
\end{tabular}

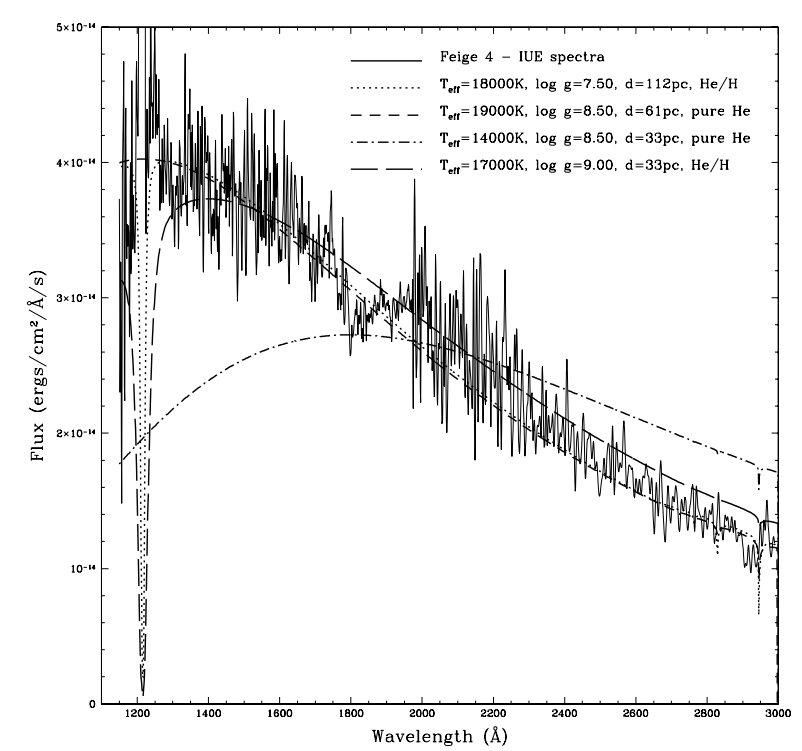

Fig. 1. IUE spectra obtained for Feige 4 (full line) compared with the best models derived leaving all parameters free for a pure He grid (dashed line) at $d=61 \mathrm{pc}$, with $T_{\text {eff }}=19000 \mathrm{~K}$ and $\log g=8.50$, for a a DB contaminated with $\mathrm{H}$ (dotted line), at $d=112 \mathrm{pc}$, with $T_{\text {eff }}=18000 \mathrm{~K}$ and $\log g=7.50$. Using the parallax distance, $(d=$ $33 \mathrm{pc}$ ) for pure He grid (dotted-dashed line), the best solution is for $T_{\text {eff }}=14000 \mathrm{~K}$ and $\log g=8.50$, and for a DB contaminated with $\mathrm{H}$ (long dashed line), the atmospheric parameters are $T_{\text {eff }}=17000 \mathrm{~K}$ and $\log g=9.00$.

April/May 2000 and the 1.0-m telescope in December 2001. At both telescopes, a high-speed CCD photometer (O'Donoghue 1995) was employed. It was operated in full-frame mode on the 0.75 -m telescope with 20 -s integrations and 3-4 s readout during the measurements in 2000, but in frame-transfer mode with 10 -s integrations in 2001. At OPD, we used the 1.6-m telescope in 1986, with a single channel photometer and 5-s integration time. We also observed at OPD in 2004, using the 0.6-m telescope and CCD 101, with 30-s integration, and 7-8 s readout. No filters were used in order to maximize the received light and considering that g-mode pulsations should have the same phase at different wavelengths (e.g. Kepler et al. 2000). We show the observing log in Table 5.

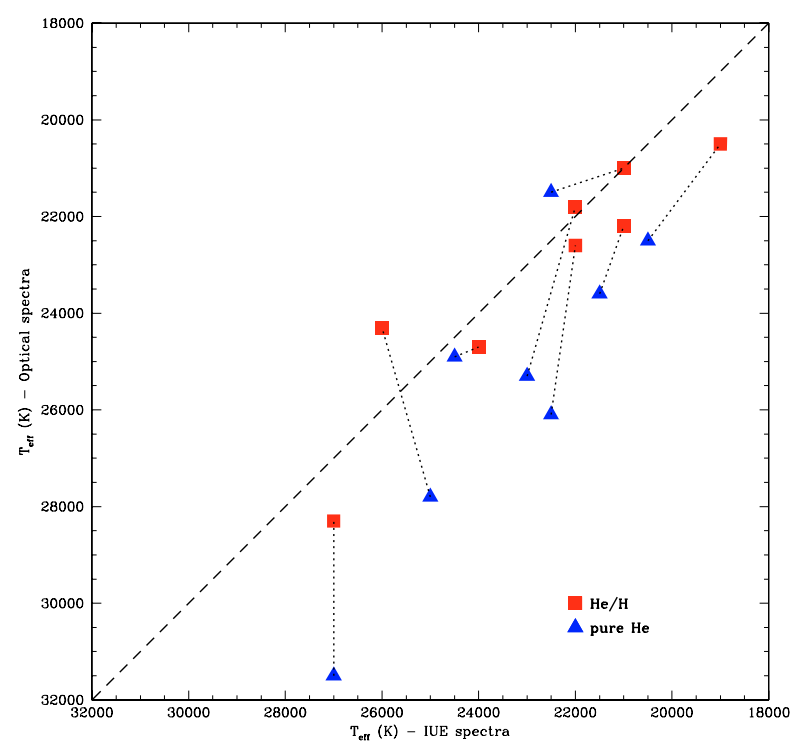

Fig. 2. Comparison between the UV ( $x$-axis) and optical ( $y$-axis) determinations for $T_{\text {eff }}$ using pure He models (blue triangles) and DB models contaminated with $\mathrm{H}$ (red squares). The dotted lines correspond to the same star. The dashed line delineates 1:1 correspondence between UV and optical spectra.

We reduced the SAAO CCD data with the standard software for this instrument, and carried out photometry by using the program MOMF (Kjeldsen \& Frandsen 1992) which uses a combined approach of PSF fitting photometry and aperture corrections on the star-subtracted frames, giving optimal results. Fourier amplitude spectra of the resulting light curves are shown in Fig. 3. For the OPD runs, the detection limits are $3 \mathrm{mma}$ for BPM 17088 and GD 270-124, $2 \mathrm{mma}$ for BPM 17731, and 1.4 mma for L 7-44.

All the seventeen stars are constant within our detection limit. The detection limits are satisfactory for all stars except PG 0949+094, WD 1415+234 (run terminated by cloud) and PG 2234+064, which should be re-observed. WD 1445+152 may also require some additional observations; the highest peak in its amplitude spectrum is somewhat outside the typical range for pulsating white dwarf stars 
Table 5. Journal of observations. $\Delta T$ is the length of the corresponding observing run.

\begin{tabular}{cccc}
\hline \hline Star & Run start (UT) & $\Delta T(\mathrm{~h})$ & \# points \\
\hline BPM 17088 & $09 / 09 / 86,05: 18$ & 1.24 & 890 \\
BPM 17731 & $11 / 09 / 86,04: 11$ & 3.17 & 2282 \\
GD 270-124 & $31 / 10 / 86,22: 54$ & 3.37 & 2423 \\
WD 0853+163 & 26/04/00, 17:46 & 1.13 & 173 \\
WD 1311+129 & $27 / 04 / 00,21: 23$ & 1.51 & 230 \\
PG 1445+152 & $28 / 04 / 00,21: 18$ & 1.33 & 195 \\
PG 0949+094 & $29 / 04 / 00,17: 00$ & 1.25 & 193 \\
PG 1026-056 & $29 / 04 / 00,18: 19$ & 1.15 & 175 \\
L 151-81A & $29 / 04 / 00,23: 53$ & 1.61 & 250 \\
WD 1134+073 & $30 / 04 / 00,18: 15$ & 1.41 & 213 \\
WD 1332+162 & $01 / 05 / 00,18: 29$ & 1.79 & 260 \\
WD 1336+123 & $01 / 05 / 00,20: 19$ & 1.43 & 222 \\
WD 1444-096 & $01 / 05 / 00,21: 48$ & 1.10 & 168 \\
WD 1415+234 & $01 / 05 / 00,22: 57$ & 0.57 & 88 \\
PG 2354+159 & $16 / 12 / 01,18: 38$ & 1.07 & 386 \\
PG 2234+064 & $17 / 12 / 01,18: 57$ & 0.91 & 326 \\
L 7-44 & $15 / 08 / 04,00: 33$ & 2.61 & 233 \\
\hline
\end{tabular}

Table 6. Variability classification of the DB stars in our sample. The $\mathrm{V}$ is used for variables, NV for non-variables and NO for not observed for variability.

\begin{tabular}{l|l}
\hline \hline & Stars \\
\hline V & PG 1115+158, PG 1351+489, PG 1456+103, \\
& GD 358, PG 1654+160 \\
NV & Feige 4, G270-124, PG 0112+104, GD 40, \\
& BPM 17731, Ton 10, PG 0853+163, GD 303, \\
& PG 1311+129, GD 325, PG 1445+152, G 256-18, \\
& GD 190, L 7-44, GD 378, G 26-10, LTT 9031 \\
& BPM 17088 \\
NO 226-32, GD 408, BPM 18164, L 748-70, & PG 0948+013, PG 1149-133, PG 1326-037, \\
& PG 1411+218, G 200-39, L 1537-31, BPM 26944 \\
\hline
\end{tabular}

but we cannot rule out that it is intrinsic to the star from the present data. We also note that we could not detect variability of the DA white dwarf L 151-81B, but our detection limit $(\sim 8 \mathrm{mma})$ is poor. On the other hand, we suspect that the star 2MASS 14581310-6317340, ( $\sim 8$ arcsec East of $\mathrm{L} 151-81 \mathrm{AB})$ is a $\delta$ Scuti star, with a 1.3 -h period and $23 \mathrm{mmag}$ semi-amplitude.

The variability classification of DB stars is shown in Table 6, where V is used for variables, NV for non-variables, and NO for not observed for variability reported.

Having derived the physical parameters from ultraviolet spectra, and the atmosphere composition for thirteen stars, we are ready to determine the DB instability strip for this homogeneous sample. In Figs. 4 and 5, we show the final determination

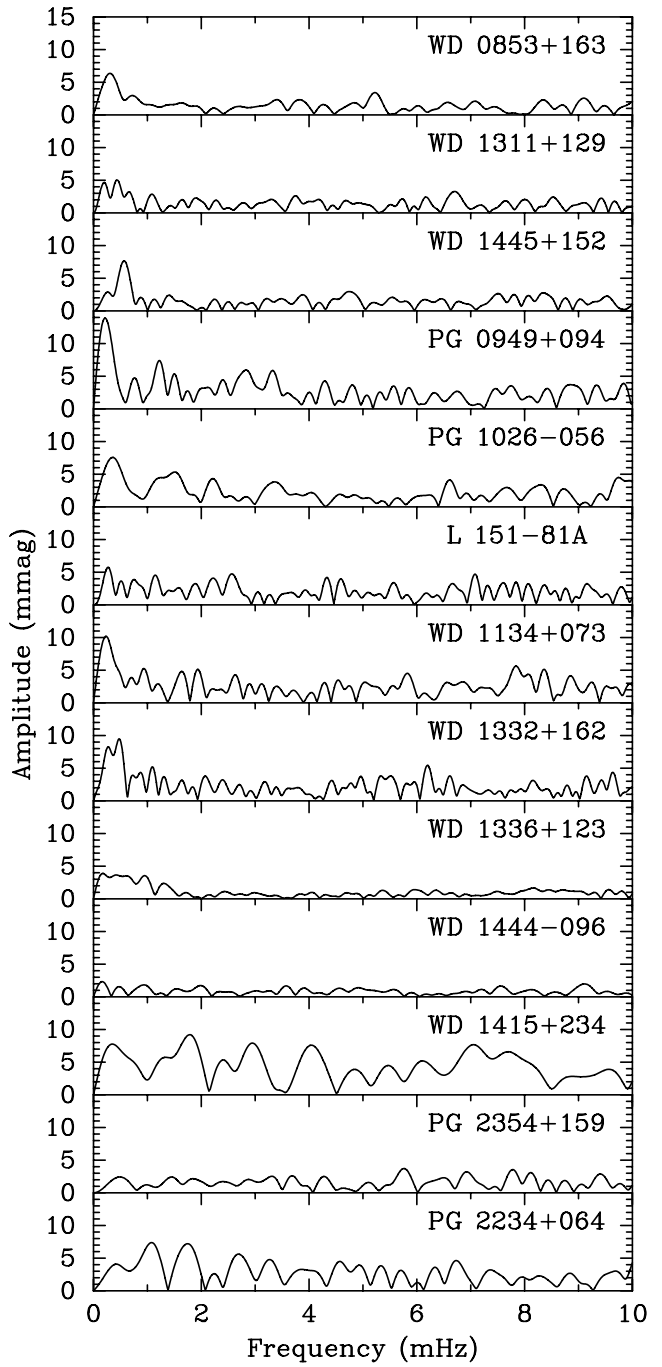

Fig. 3. Fourier amplitude spectra of the null results of a search for pulsation among DB white dwarf stars.

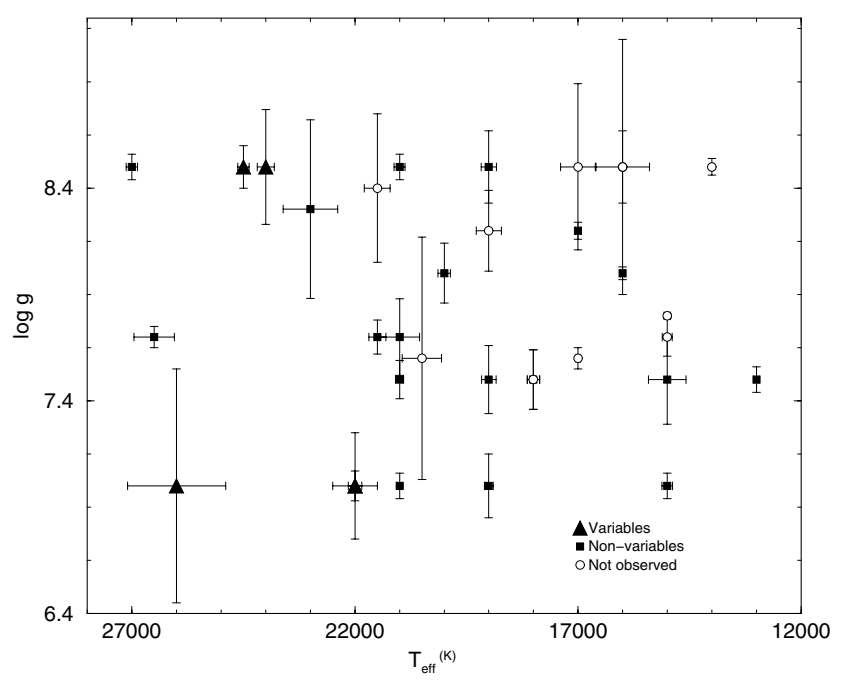

Fig. 4. DB instability strip using pure He models for the stars for which we cannot determine atmosphere composition: variables (filled triangle), non-variables (filled squares), and not observed for variability (open circles). There are 2 stars close to the instability strip that have not been observed for variability. 


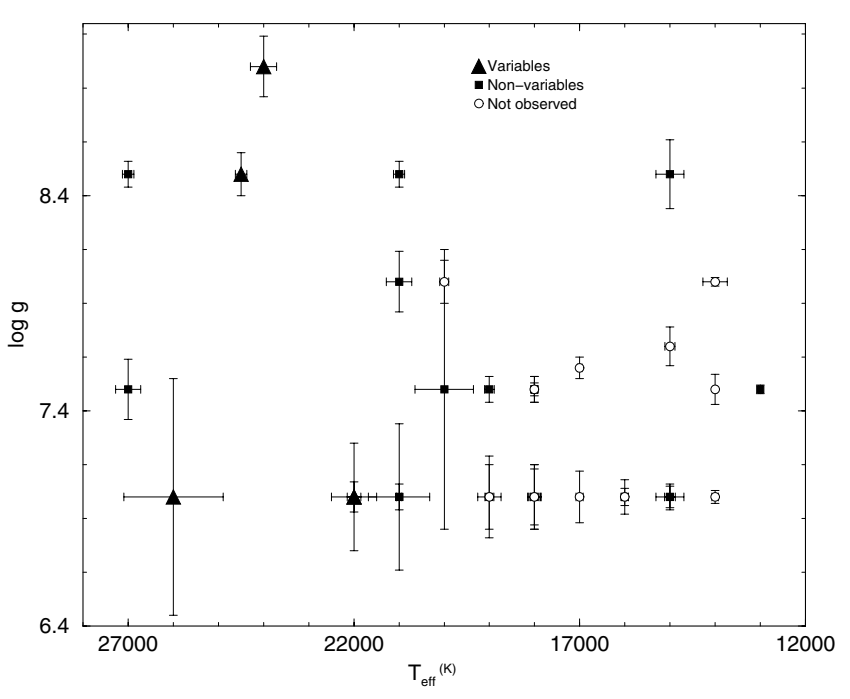

Fig. 5. DB instability strip using $\mathrm{H}$ contaminated $\mathrm{He}$ models for the stars for which we cannot determine atmosphere composition: variables (filled triangle), non-variables (filled squares), and not observed for variability (open circles). There are 2 stars close to the instability strip that have not been observed for variability.

for $T_{\text {eff }}$ and $\log g$ for variables (filled triangle), non-variables (filled squares), and so far not observed by time series photometry (open circles) DB stars. This diagram shows that DBs pulsate in a well-defined temperature range, from $26000 \geq$ $T_{\text {eff }} \geq 22000 \mathrm{~K}$. For the stars which we could not determine their atmosphere composition, we used both pure He models and $\mathrm{He} / \mathrm{H}$ models, respectively. There is a $97 \%$ chance that the DB instability strip contains only variable stars. Even if the error bars in $T_{\text {eff }}$ were three times larger, there is only a $4 \%$ probability of contamination. This probability was calculated by adding the probability of all variables that fall inside the instability strip and all non-variables outside, using Gaussian distributions for our $T_{\text {eff }}$ determinations only (no consideration for $\log g$ ). There are still stars close to the instability strip that have not been searched to our knowledge for variability, and which are crucial for the study of the instability strip.

However, Beauchamp et al.'s (1999) optical spectra fitting found non-variables inside the instability strip. In this sense, for a true determination of the DB instability strip it is necessary to fit the optical and UV spectra simultaneously, to analyze the possible differences to convection prescription.

\section{Concluding remarks}

We used model atmospheres with ML2/ $\alpha=0.6$ to derive atmospheric parameters $\left(T_{\text {eff }}\right.$ and $\log g$ ) and distances for thirty four DB stars with available IUE re-calibrated spectra. Our model grid fit well the spectra. Another important conclusion is that atmospheric contamination with $\mathrm{H}$ is not directly proportional to $T_{\text {eff }}$ for DB stars, based on our determination for eleven stars, which has been a suggestion to explain the DB gap by convection dragging $\mathrm{H}$ upwards. We also find no DB stars inside the DB gap.

Acknowledgements. Financial support: CAPES/UT grant, CNPq fellowship.

\section{References}

Althaus, L. G., \& Benvenuto, O. G. 1997, ApJ, 477, 313

Althaus, L. G., Serenelli, A. M., Panei, J. A., et al. 2005, A\&A, 435, 631

Beauchamp, A., et al. 1999, ApJ, 516, 887

Bergeron, P., Saumon, D., \& Wesemael, F. 1995, ApJ, 443, 764

Bergeron, P., Leggett, S. K., \& Ruiz, M. T. 2001, ApJS, 133, 413

Corsico, A. H., \& Althaus, L. G. 2004, A\&A, 428, 159

Finley, D. S., Koester, D., \& Basri, G. 1997, ApJ, 488, 375

Hansen, B., \& Liebert, J. 2003, ARA\&A, 41, 465

Holberg, J. B., Barstow, M. A., \& Burleigh, M. R. 2003, ApJS, 147, 145

Kepler, S. O., Robinson, E. L., Koester, D., et al. 2000, ApJ, 539, 379

Kjeldsen, H., \& Frandsen, S. 1992, PASP, 104, 413

Koester, D., \& Vauclair, G. 1997, White dwarfs, ASSL 214, 429

Metcalfe, T. S. 2003, ApJ, 587, L43

Metcalfe, T. S. 2005, MNRAS, 363, L86

Montgomery, M. H., \& Winget, D. E. 2000, Baltic Astron., 9, 23

Nitta, A., et al. 2005, in prep.

O’Donoghue, D. 1995, Baltic Astronomy, 4, 519

Provencal, J. L., Shipman, H. L., Thejll, P., \& Vennes, S. 2000, ApJ, 542,1041

Robinson, E. L., \& Winget, D. E. 1983, PASP, 95, 386

van Altena, W. F., Lee, J. T., \& Hoffleit, E. D. 2001, VizieR Online Data Catalog, 1238, 0

Winget, D. E., Robinson, E. L., Nather, R. D., Fontaine, G. 1982, ApJ, 262, L11

Winget, D. E., Sullivan, D. J., Metcalfe, T. S., Kawaler, S. D., \& Montgomery, M. H. 2004, ApJ, 602, L109

Wolff, B., Koester, D., Montgomery, M. H., \& Winget, D. E. 2002, A\&A, 388, 320 